\title{
On-chip low-profile nano-horn metal-clad optical cavity with much improved performance
}

\author{
Zheng Li, and Hyuck Choo \\ Department of Electrical Engineering, California Institute of Technology, Pasadena, CA 91125 \\ zli@,caltech.edu,hchoo@,caltech.edu
}

\begin{abstract}
We propose an on-chip nano-horm-shaped metal-clad cavity. The proposed device is $0.8 \mu \mathrm{m}$ in height-half the size of the previously reported devices-- and achieves the quality factor of 1000 and effective volume of $0.31(\lambda / n)^{3}$.
\end{abstract}

OCIS codes: (250.5403) Plasmonics; (230.5750) Resonators; (250.5300) Photonic Integrated Circuit

\section{Introduction}

Recent studies have suggested that the use of metal-clad cavities can be advantageous for on-chip applications due to their ultra-small fingerprint and efficient coupling with integrated waveguides [1,2]. To accomplish high quality factors over 1000, both the metallic absorption and the leakage into the substrate must be effectively suppressed. However, due to the low index contrast among III-V materials, it is difficult to meet these requirements in cavities with vertical profiles less than $1.5 \mu \mathrm{m}$ due to relatively short cutoff regions. Here, we propose a novel nano-horn metal-clad optical cavity that is approximately $0.8 \mu \mathrm{m}$ in height yet achieves a $Q$ factor of $\sim 1000$ (or about six times higher than those of previously reported approaches) and the effective volume of $0.31(\lambda / n)^{3}$. For simulation, we used full 3D simulations as well as our highly time-efficient yet accurate 1D model, which is about 100 times faster than full 3D simulations [3].

\section{Performance of nano-horn optical cavity}

The proposed nano-horn metal-clad cavity is shown in Fig. 1(a). The cavity consists of (a) a subwavelength-scale rectangular InGaAsP optical cavity with conducting InP posts; (b) a linearly tapering low-index $\mathrm{SiO}_{2}$ cladding layer that covers the cavity core from the top to bottom; and (c) a uniform metallic layer on the oxide cladding layer. Intuitively, this horn-like geometry pushes the major part of the mode into the horizontally wider region in the upper part of the cavity, and, in return, this results in a longer effective cut-off region. The $|E|^{2}$ distribution of the $z$ - $x$ cross section (Fig. 1(b)) shows that the field profile does not come in contact with the sloped metallic walls yet fits in the tapering geometry of the cladding layer. The wider horizontal space that accommodates the mode in the cavity and a longer cut-off region between the mode and the substrate reduce the optical absorption from the metallic walls and the radiation loss to the substrates, respectively, and further boost the Q factor. Laterally, the mode retains its donut shape along the $x-y$ cross-sectional planes (Fig. 1 (b) crl-cr3), which also agrees with the 2D modal calculation in Fig. 1(c).
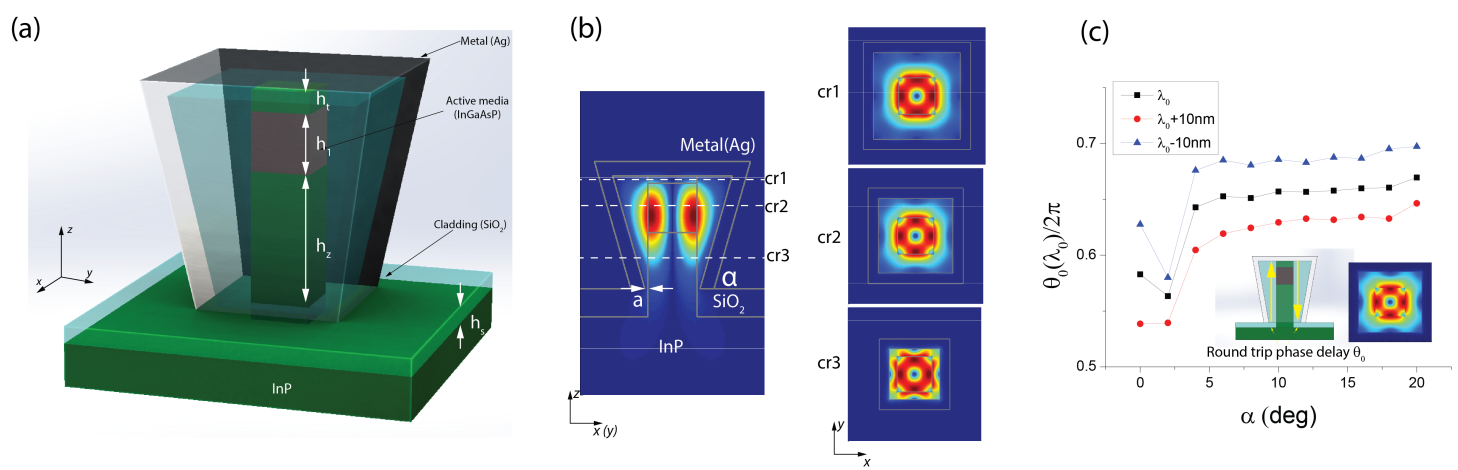

Fig 1. (a) Schematic illustration of proposed nano-horn metallic semiconductor nanocavity: the semiconductor (InGaAs) cavity has the dimensions of $350 \mathrm{~nm} \times 350 \mathrm{~nm} \times 350 \mathrm{~nm}$. InP posts with the same cross-sectional dimensions are connected to the metal clad and the substrate. (b) Cross-sectional electric field-intensity distributions of the mode supported by this cavity along the $x-z$ plane and the $x-y$ planes are denoted by $\mathrm{cr} 1$, cr2, and cr3. The cavity resonates at $\lambda_{0}=1494 \mathrm{~nm}$ with $h_{\mathrm{t}}: h_{1}: h_{\mathrm{z}}: h_{\mathrm{s}}=50: 350: 400: 200 \mathrm{~nm}$, the bottom clad thickness $a=20 \mathrm{~nm}$, the taper angle $\alpha=16^{\circ}$, and the metal thickness $t_{\mathrm{m}}=100 \mathrm{~nm}$. (c) Phase delay $\theta_{0}$ is calculated against the taper angle $\alpha$. The inset illustrates the calculation routine of the phase delay and the 2D transverse-electric (TE) fundamental waveguide mode supported by the structure very similar to cr1-3 [4]. 
We first performed full 3D finite difference time domain (FDTD) simulation for the proposed structure. In Figure 2 (a) and (b), we plot the resonant wavelength and Purcell factor for the case shown in Fig. 1(b) as a function of taper angle $\alpha$. The results confirm our previous explanations. As the taper angle $\alpha$ is increased, the low-index cladding layer becomes wider and reduces the average effective index for the 2D waveguide mode, which in turn makes the cavity resonate at a longer wavelength. Similarly, the total cavity $Q$ factor $Q_{\text {tot }}$ can be decomposed into two components: (a) the metallic absorption $Q_{\text {abs }}$ and (b) the radiation loss to the substrate $Q_{\text {rad }}$ or $Q_{\text {tot }}^{-1}=Q_{\mathrm{rad}}^{-1}+Q_{\mathrm{abs}}^{-1}$. Increasing the taper angle also enhances both $Q$-factors, and in the region between $\alpha=10$ and $\alpha=16$, the Q factor reaches above $10^{3}$. Interestingly, even if the cavity size increases due to increase in $\alpha$, the effective volume itself does not increase noticeably because the electromagnetic fields are still mainly confined in the high-dielectric region. In fact, the effective volume increases at a rate slower than the cubic resonant wavelength $\lambda_{0}^{3}$ and contributes positively to the enhancement of the Purcell factor, which can reach $\sim 300$ at $\alpha \sim 15$. Choosing a thicker cladding layer ( $a=100 \mathrm{~nm}$, Fig. 1(b)) for the case with $\alpha=0$ (previously employed case) and comparing to the case with $\alpha=16^{\circ}$ in a similar wavelength domain (Fig. 2(c)) reveals that our approach improves $Q$ by a factor of $\sim 6$. Additionally, we have also observed that the size of the bottom separation layer $h_{s}$ mainly affects the $Q$-factor, not the resonant wavelength.

(a)

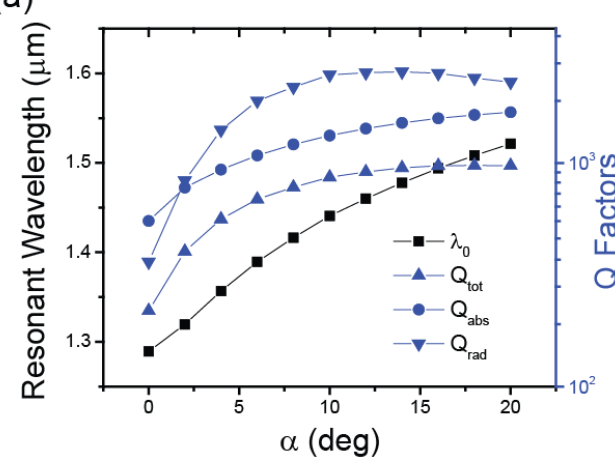

(c)

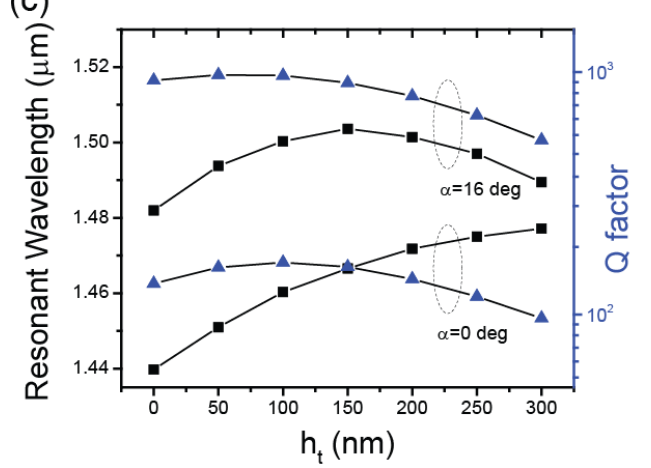

(b)

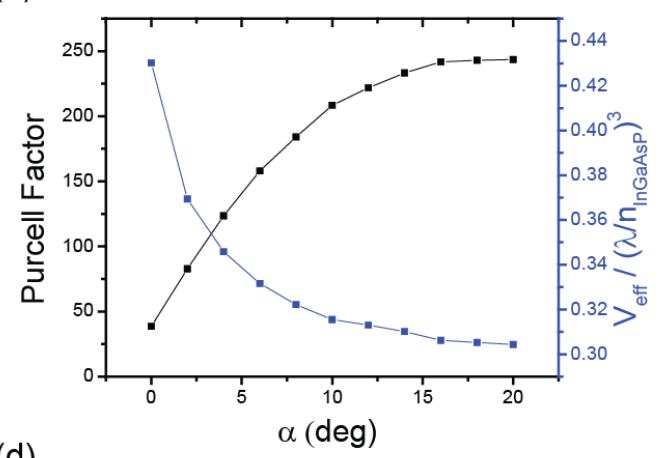

(d)

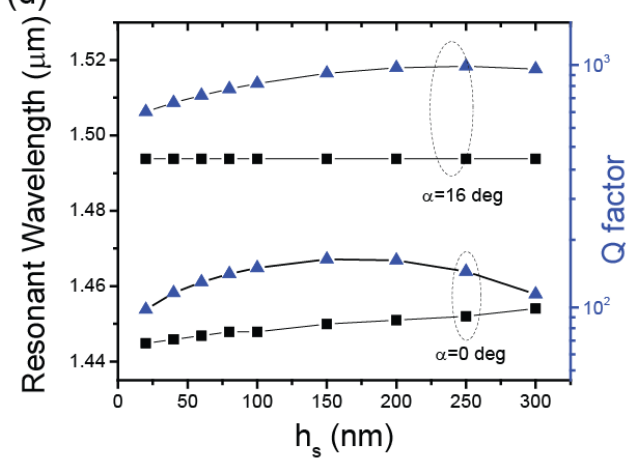

Fig 2. (a) Resonant wavelength and Q factors and (b) Purcell factor $F_{\mathrm{P}}$ and Effective volume $V_{\text {eff }}$ are plotted as functions of the taper angle $\alpha$ with the same parameter set used in Fig. 1. (b). Resonant wavelength and Q factor as functions of $h_{t}$ and $h_{s}$, defined in Fig. 1 (a) and with total cavity height $800 \mathrm{~nm}$. Here we set the bottom cladding thickness at $100 \mathrm{~nm}$ for the case with $\alpha=0$.

\section{Conclusion}

Using both full 3D FDTD simulations and computationally efficient1D semi-analytic model, we have proposed and studied the design and properties of the nano-horn metal-clad optical cavity with much improved performance. We believe that this approach could potentially lead us to ultra-compact, ultra-fast, and cost-efficient on-chip devices.

\section{References}

[1] M. T. Hill and M. J. H. Marell, "Surface-Emitting Metal Nanocavity Lasers," Advances in Optical Technologies, 2011, 314952 (2011).

[2] M. Kim, Z. Li, and H. Choo, "Engineering of metal-clad optical nanocavities to optimize coupling with integrated waveguides," Opt. Express 21, 25796-25804 (2013).

[3] Z. Li and H. Choo, "Computationally Efficient and Intuitive 1D Analytical Model for 3D Metal-Clad Nanocavities," 2013 MRS Fall Meeting, December 1-6, 2013, Boston, MA.

[4] The round trip adiabatic phase change, $\theta_{0}$, is defined as $\theta_{0}\left(\lambda_{0}\right)=4 \pi \int_{z \in \text { cavity }} n_{e f f}\left(z, \lambda_{0}\right) / \lambda_{0} d z$ for the given wavelength $\lambda_{0}$ and the effective index $n_{\text {eff }}$ of the $2 \mathrm{~d}$ fundamental TE waveguide mode. 